\title{
Influence of Magnetic Field on Detection Properties of Planar Microwave Diodes
}

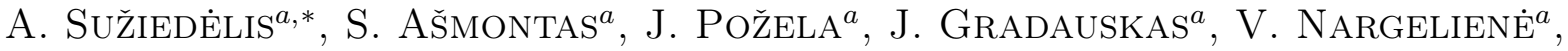

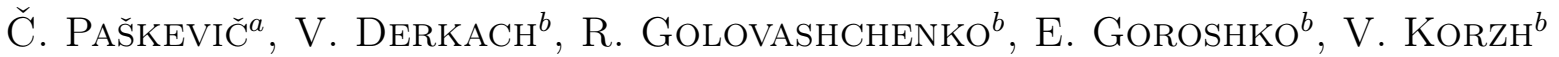 \\ AND T. ANBINDERIS ${ }^{c}$ \\ ${ }^{a}$ Semiconductor Physics Institute, Center for Physical Sciences and Technology \\ A. Goštauto 11, Vilnius, Lithuania \\ ${ }^{b}$ Usikov Institute of Radiophysics and Electronics of NAS of Ukraine \\ 12 Ac. Proskura Str., 61085 Kharkov, Ukraine \\ ${ }^{c}$ Elmika Ltd, Naugarduko Str. 41, Vilnius LT-03227, Lithuania
}

\begin{abstract}
The results of experimental investigation of detection properties of the planar microwave diodes of various configuration on DC magnetic field are presented in this paper. The detection of microwave radiation was measured at $51 \mathrm{GHz}, 72 \mathrm{GHz}$ and $144 \mathrm{GHz}$ frequencies. The magnetic field was applied in plane and perpendicularly to the plane of the diodes. The experiment was performed at room temperature. Dependence of the detected voltage of the diodes on the magnetic field had asymmetric character with respect to the polarity of the magnetic field. This fact allowed us to suspect the magnetic rectification influencing the detected voltage. Therefore, average value of the detected voltage with respect to the polarity of the applied magnetic field gives its dependence on the applied magnetic field.
\end{abstract}

PACS: 07.57.Kp, 78.70.Gq, 75.47.Pq, 73.40.Kp, 73.40.-c, 75.70.-i

\section{Introduction}

Magnetoresistance phenomena in two-dimensional electron system under influence of microwave radiation attract attention of researchers both from fundamental point of view and for application purposes [1-4]. These phenomena are related with high electron mobility in two-dimensional electron gas (2DEG) channel and cyclotron resonance that usually are observed at cryogenic temperatures. We proposed to use semiconductor structures with constricted sheets of electrons for broad-band microwave detection exploring non-uniform carrier heating in microwave electric fields [5]. Voltage sensitivity of these detectors is inversely proportional to the thickness of the electron sheet. Therefore, various semiconductor structures, starting from heavily doped $n$-GaAs layers of submicrometric thickness [6] down to the delta-doped [7] and modulation doped semiconductor structures [8] of nanometric thickness were used for the fabrication of microwave diodes. The voltage sensitivity of the diodes increased with crystal lattice temperature decrease, however at room temperature the microwave diodes sensed the microwave power as well. Since the magnetic field

\footnotetext{
* corresponding author; e-mail: as@pfi.lt
}

changes the resistance of the two-dimensional electron system, we have investigated the detection properties of various planar microwave diodes whose operation is based on the carrier heating phenomena in external magnetic field. Experimental results of the detected voltage on magnetic field at room temperature are presented in this paper.

\section{Samples and experimental}

Samples under test were fabricated on the basis of molecular beam epitaxy (MBE) grown semiconductor structures: (i) heavily doped $n^{+}$-GaAs layer of $100 \mathrm{~nm}$ thickness grown onto $i$-GaAs buffer layer. We refer to this structure as submicrometric structure and shall denote as SS; (ii) five delta-doped layers separated by $25 \mathrm{~nm}$ thick $i$-GaAs layers. We call this structure as nanometric one and denote as NS; (iii) heavily doped $n^{+}-\mathrm{Al}_{0.3} \mathrm{Ga}_{0.7} \mathrm{As}$ layer of $100 \mathrm{~nm}$ thickness grown onto $i$-GaAs buffer layer. We call this structure as modulation doped structure and denote it as MDS. The microwave diodes were fabricated etching semiconductor mesas of symmetrically and asymmetrically constricted shape and evaporating $\mathrm{Ge} / \mathrm{Ni} / \mathrm{Au}$ metals onto the etched mesas. After the rapid annealing of the evaporated metals at $430{ }^{\circ} \mathrm{C}$ temperature in forming gas atmosphere, the ohmic con- 
tacts to semiconductor were made. Specific contact resistance was controlled using transmission line method. The value of the specific contact resistance did not exceed $1 \Omega \mathrm{mm}$. Then thin polyimide film caped the face side of the semiconductor substrate. Semiconductor substrate was thinned down using mechanical polishing and wet etching to approximately $5 \mu \mathrm{m}$. This design of the microwave diodes is up for the high frequency application because the thin semiconductor mesa onto a dielectric film with low dielectric permittivity represents the quasi free-standing structure. The microwave diodes with symmetrically (SD) and asymmetrically (AD) shaped $n^{+}-n$ junction are depicted in Fig. 1. The detection of MW radiation was measured at $51 \mathrm{GHz}, 72 \mathrm{GHz}$ and $144 \mathrm{GHz}$ frequencies. The magnetic field was applied in plane $\left(H_{\|}\right)$ and perpendicularly $\left(H_{\perp}\right)$ to the plane of the diodes. The experiment was performed at room temperature.

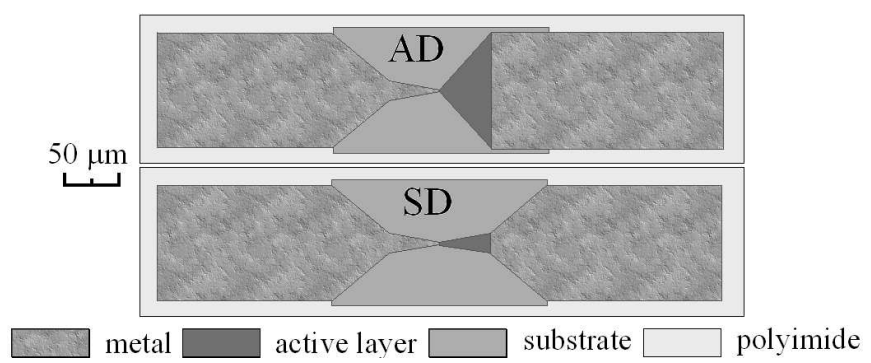

Fig. 1. Schematic view of the planar microwave diodes.

\section{Experimental results and discussion}

Dependence of the detected voltage of the asymmetrically shaped diodes on the magnetic field had asymmetric character with respect to the polarity of the magnetic field. Figure 2 shows the dependence of the detected voltage $U_{\mathrm{d}}$ on external magnetic field $H$ for the diode based on NS. This fact allows us to suspect the magnetic rectification influencing the detected voltage [3]. Therefore, average value of the detected voltage with respect to the polarity of the applied magnetic field gives its dependence on the applied magnetic field. Figure 3 depicts the influence of external magnetic field on averaged relative voltage sensitivity $S(H) / S(0)$ of MW diode on the base of asymmetrically shaped GaAs delta doped structure, where $S(H)=U_{\mathrm{d}} / P$ and $P$ is MW power in the waveguide.

MW diodes on the base of modulation doped structure should reveal higher voltage sensitivity due to the presence of two-dimensional electron gas in this structure. The dependence of detected voltage on magnetic field of the asymmetrically shaped MDS are shown in Fig. 4. Here we also can see the magnetic rectification because the detected voltage depends on the polarity of the magnetic field. However, the dependence of the detected voltage on magnetic field is different for the NS and MDS diodes when we consider the different orientation of the diodes with respect to the magnetic field.

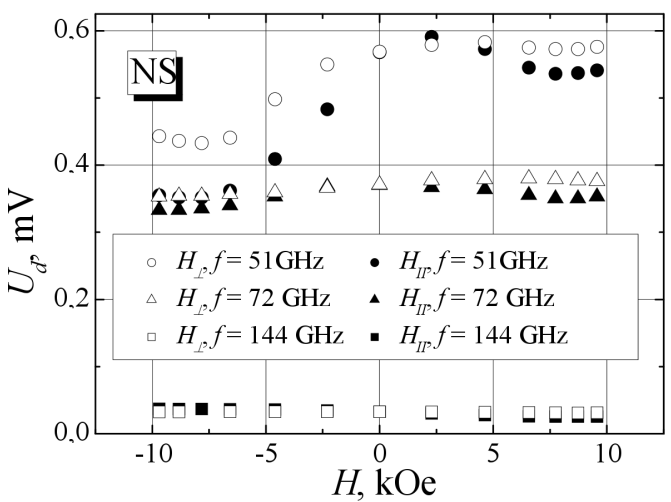

Fig. 2. The dependence of detected voltage on magnetic field for the MW diode based on nanometric structure.

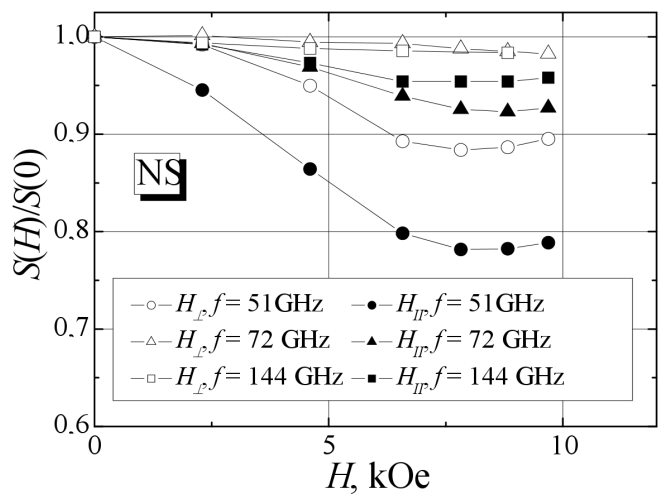

Fig. 3. Averaged relative voltage sensitivity $S(H) / S(0)$ of the NS MW diode versus external magnetic field.

In case of the NS diodes the detected voltage is more sensitive to the magnetic field when the diode is parallel to the magnetic field and, contrary, in case of the MDS diodes the detected voltage is more sensitive to the magnetic field when the diode is perpendicular to the magnetic field. This dependence on the orientation of the diode is clearly seen in Fig. 5, where the dependences of averaged relative voltage sensitivity on magnetic field are presented. It is worth to note the dependence of the voltage sensitivity on frequency: the value of the voltage sensitivity diminishes with frequency increase as well as the influence of the magnetic field is less at higher frequencies.

The influence of magnetic field on the detected voltage should depend on shape of the MW diodes. The dependence of relative voltage sensitivity on magnetic field are presented in Fig. 6 for the asymmetrically and symmetrically shaped diodes on the base of submicrometric structures. It can be seen that the voltage sensitivity of the asymmetrically shaped diode is more sensitive to magnetic field than that of the symmetrically shaped submicrometric structure. It is of interest to compare the 


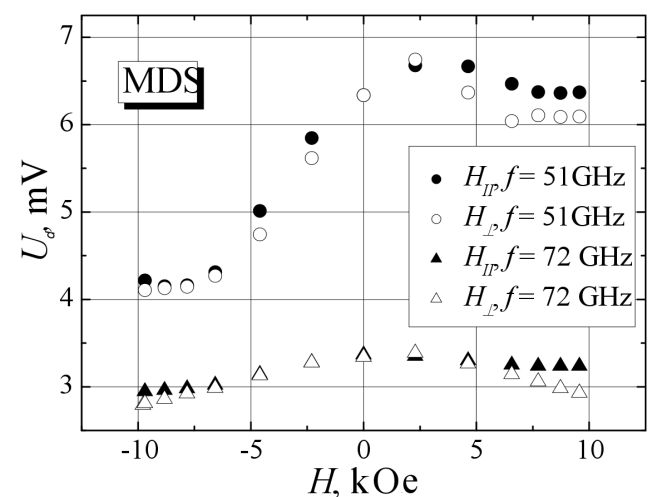

Fig. 4. The dependence of detected voltage on magnetic field for the MW diode based on modulation doped structure.

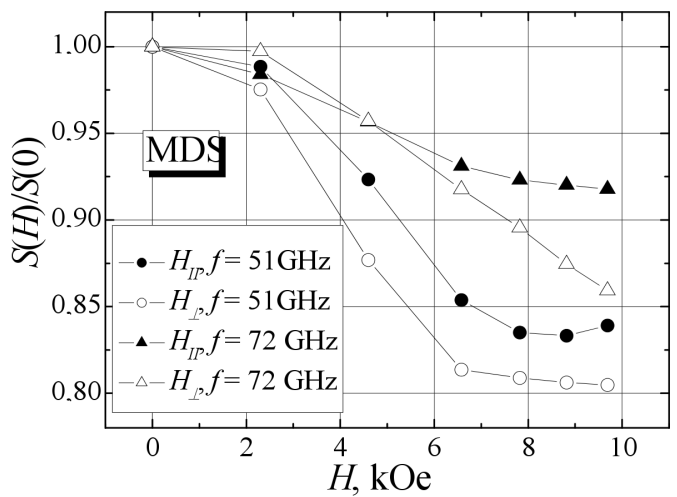

Fig. 5. Averaged relative voltage sensitivity $S(H) / S(0)$ of the MDS MW diode versus external magnetic field.

magnetic field influence onto detected voltage depending on shape of microwave diodes on the base of the modulation doped structure when electrons are collected in two-dimensional sheet of nanometric size. The dependence of averaged relative voltage sensitivity on magnetic field of the AD and SD on the base of MDS are shown in Fig. 7. In case of the MW diodes on the base of the MDS the influence of magnetic field is more pronounced for the asymmetrically shaped diodes. On the other hand, the voltage sensitivity of the microwave diodes on the base of modulation doped structure is less sensitive to magnetic field with respect to the orientation of the diode in regard to the magnetic field.

\section{Conclusions}

Investigation of detection properties of planar microwave diodes on the base of various semiconductor structures with two-dimensional or quasi-two-dimensional electron gas allows us to conclude:

- the detected voltage depends on polarity of the external magnetic field that evidences magnetorectification of microwave signal;

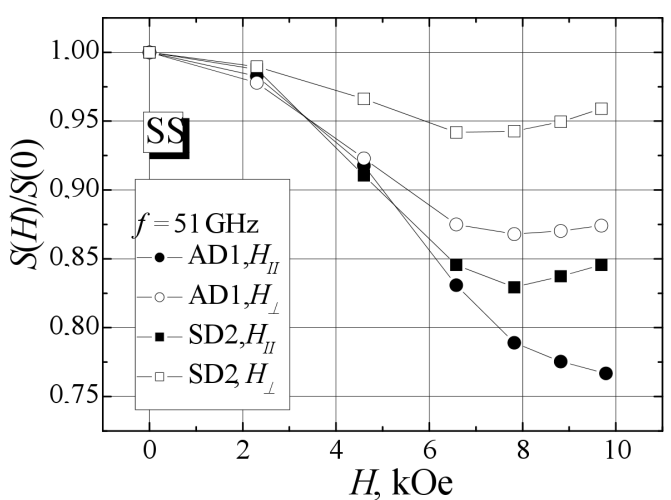

Fig. 6. Averaged relative voltage sensitivity $S(H) / S(0)$ of the SS MW diode of different shape versus external magnetic field.

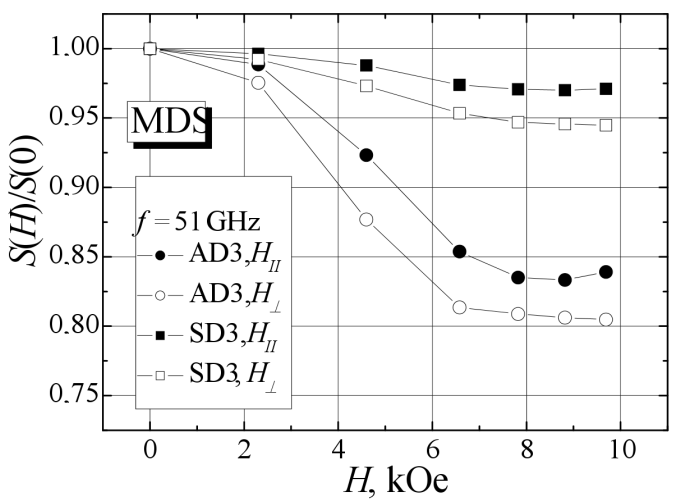

Fig. 7. Averaged relative voltage sensitivity $S(H) / S(0)$ of the MDS MW diode of different shape versus external magnetic field.

- averaged relative voltage sensitivity of the MW diodes decreases with microwave frequency increase. This experimental fact supports the presumption concerning rectification nature of the detected voltage;

- different dependences of voltage sensitivity on magnetic field were observed for the MW diodes on the base of semiconductor structures with directly doped layers and modulation doped structures for different orientation of the diodes with respect to external magnetic field;

- stronger influence on voltage sensitivity of the planar microwave diodes was observed in case of asymmetrically shaped semiconductor structures.

\section{Acknowledgments}

This work was supported by Science and Research Council of Lithuania under contracts No. TAP-20/2010 and No. TAP-56/2010 as well as by Ministry of Education and Science of Ukraine under contract M/141-2009. 
Samples were fabricated at Braun Submicron Research Center under EU project RITA-CT-2003-506095.

\section{References}

[1] I.V. Kukushkin, J.H. Smet, V.I. Fal'ko, K. von Klitzing, K. Eber, Phys. Rev. B 66, 121306(R) (2002).

[2] A.E. Patrakov, I.I. Lyapilin, Phys. Rev. B 75, 155320 (2007).

[3] S. Kataoka, H. Naito, Solid-State Electron. 9, 459 (1966).

[4] S.I. Dorozhkin, J.H. Smet, K. von Klitzing, L.N. Pfeiffer, K.W. West, JETP Lett. 86, 543 (2007).
[5] S. Ašmontas, A. Sužiedèlis, Int. J. Infrared Millimeter Waves 15, 525 (1994).

[6] S. Ašmontas, J. Gradauskas, A. Kozič, H. Shtrikmann, A. Sužiedèlis, Acta Phys. Pol. A 107, 147 (2005).

[7] S. Ašmontas, V. Kazlauskaitè, A. Sužiedèlis, J. Gradauskas, V. Derkach, J. Phys., Conf. Series 193, 012120 (2009).

[8] A. Juozapavičius, L. Ardaravičius, A. Sužiedèlis, A. Kozič, J. Gradauskas, J. Kundrotas, D. Seliuta, E. Sirmulis, S. Ašmontas, G. Valušis, H.G. Roskos, K. Köhler, Semicond. Sci. Technol. 19, S436 (2004). 